\title{
The role of carbon nanotubes in improving thermal stability of polymeric fluids; experimental and modeling
}

Mohamad Amin Halali ${ }^{~}$, Cyrus Ghotbi ${ }^{1 *}$, Kourosh Tahmasbi ${ }^{2}$, Mohammad Hossein Ghazanfari ${ }^{1}$

${ }^{\prime}$ Chemical and Petroleum Engineering Department, Sharif University of Technology, Tehran, Iran

${ }^{2}$ Pars Drilling Fluids Company, Tehran, Iran

\footnotetext{
* Corresponding author:

Email Address: Ghotbi@sharif.edu (Cyrus Ghotbi)
} 


\section{Appendix A. Statistical equations}

C.1. Average Correlation Factor (R2)

$$
R^{2}=1-\frac{\sum\left(\left(\frac{d P}{d x}\right)_{\text {pred }^{-}}\left(\frac{d P}{d x}\right)_{\text {exp }}\right)^{2}}{\sum\left(\left(\frac{d P}{d x}\right)_{\text {pred }}-\left(\frac{d P}{d x}\right)_{e x p}\right)^{2}}
$$

C.2. Root Mean Square Error (RMSE)

$$
R M S E=\sqrt{\frac{1}{N} \sum\left(\left(\frac{d P}{d x}\right)_{p r e d}-\left(\frac{d P}{d x}\right)_{e x p}\right)^{2}}
$$

